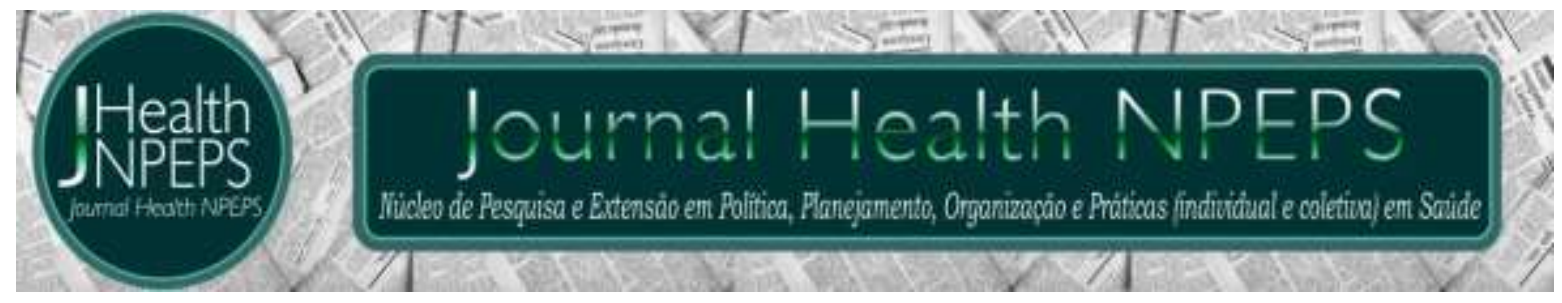

http://dx.doi.org/10.30681/252610103415

ARTIGO ORIGINAL

\title{
Perfil de saúde de mulheres atendidas em estratégias saúde da família em Mato Grosso
}

\author{
Health profile of women assisted in family health strategies in Mato Grosso \\ Perfil de salud de mujeres atendidas en estrategias salud de la familia en \\ Mato Grosso
}

\section{Nirlande Rodrigues da Silva ${ }^{1}$, Heloísa Rodrigues Xavier ${ }^{2}$, Thiago Lara da Rocha ${ }^{3}$, Veridiana Lourenço Tavares Santos ${ }^{4}$, Magda de Mattos ${ }^{5}$, Débora Aparecida da Silva Santos ${ }^{6}$, Aristides José da Silva Júnior ${ }^{7}$}

\section{RESUMO}

Objetivo: descrever o perfil de saúde de mulheres atendidas em estratégias saúde da família em Mato Grosso. Método: estudo transversal, descritivo e com abordagem quantitativa, realizada com 261 mulheres. A coleta de dados ocorreu no período de setembro a dezembro de 2017, por meio da aplicação de questionário estruturado, que abrangeu características sóciodemográficas, estilo de vida, nutrição e fatores de riscos específicos. Para análise de dados, utilizou-se estatística descritiva, Teste de Mann Whitney e ANOVA, considerando nível de significância de $5 \%$, que foi realizado através do programa Epi Info. Resultados: houve predomínio na faixa etária entre 25 a 44 anos $(34,1 \%)$. A realização de atividade física foi referida por $36,4 \%$, prática do tabagismo por $10,3 \%$ e a ingestão de bebidas alcoólicas por $20,3 \%$ das mulheres. A medida da circunferência abdominal encontrava-se inadequada em $47,9 \%$ das mulheres. Conclusão: as condições de saúde das participantes, relacionadas principalmente aos hábitos de

\footnotetext{
${ }^{1}$ Graduanda em Enfermagem. Universidade Federal de Rondonópolis (UFR). Rondonópolis, MT, Brasil. E-mail: nirlandeenf@gmail.com ORCID ID: https://orcid.org/0000-0003-0464-8296 Autor principal - Endereço para correspondência: Rua A 129, no 915. Bairro Sagrada Família, Rondonópolis/MT, CEP: 78735-024.

${ }^{2}$ Graduanda em Medicina. Universidade Federal de Rondonópolis (UFR). Rondonópolis, MT, Brasil. E-mail: heloisar.xavier@hotmail.com ORCID ID: https://orcid.org/0000-0001-6471-916X

${ }^{3}$ Graduando em Medicina. Universidade Federal de Rondonópolis (UFR). Rondonópolis, MT, Brasil. E-mail: thiagorocha11@hotmail.com ORCID ID: https://orcid.org/0000-0003-4433-7898

${ }^{4}$ Graduanda em Medicina. Universidade Federal de Rondonópolis (UFR). Rondonópolis, MT, Brasil. E-mail: veridiana tavares@hotmail.com ORCID ID: https://orcid.org/0000-0001-7627-8963

${ }^{5}$ Enfermeira. Doutora em Educação. Docente Adjunta da Universidade Federal de Rondonópolis (UFR). Rondonópolis, MT, Brasil Brasil. E-mail: magda roo@hotmail.com ORCID ID: https://orcid.org/0000-0001-8330-1084

${ }^{6}$ Enfermeira. Doutora em Recursos Naturais. Docente Adjunta da Universidade Federal de Rondonópolis (UFR). Rondonópolis, MT, Brasil. E-mail: deboraassantos@hotmail.com ORCID ID: https://orcid.org/0000-0003-1862-7883 ${ }^{7}$ Enfermeiro. Doutor em Educação. Docente Adjunto da Universidade Federal de Rondonópolis (UFR). Rondonópolis, MT, Brasil. E-mail: aristides@ufmt.br ORCID ID: https://orcid.org/0000-0002-1707-6664
} 
vida, caracterizaram um perfil de risco para desenvolvimento de doenças crônicas não transmissíveis. Conhecer o perfil das mulheres atendidas na ESF contribui para o planejamento de estratégias educativas a esta população.

Descritores: Perfil de Saúde; Saúde da Mulher; Estratégia Saúde da Família.

\section{ABSTRACT}

Objective: to describe the health profile of women assisted in family health strategies (FHS) in Mato Grosso. Method: a cross-sectional, descriptive study with a quantitative approach was performed with 261 women. Data collection took place in the period from September to December 2017, through the application of a structured questionnaire, which covered sociodemographic characteristics, lifestyle, nutrition and specific risk factors. For data analysis, descriptive statistics, Mann Whitney test and ANOVA were carried out considering a level of significance of 5\%, which was performed through the Epi Info program. Results: there was predominance in the age group between 25 and 44 years (34.1\%). Physical activity was reported by $36.4 \%$, smoking practice by $10.3 \%$ and alcoholic beverages by $20.3 \%$ of women. Measurement of abdominal circumference was inadequate in 47.9\% of women. Conclusion: the health conditions of the participants, related mainly to the habits of life, characterized a risk profile for the development of chronic noncommunicable diseases. Knowing the profile of the women attended at the FHS contributes to the planning of educational strategies for this population.

Descriptors: Health Profile; Women's Health; Family Health Strategy.

\section{RESUMEN}

Objetivo: describir el perfil de salud de mujeres atendidas en estrategias salud de la familia en Mato Grosso. Método: estudio transversal, descriptivo y con abordaje cuantitativo, realizado con 261 mujeres. La recolección de datos ocurrió en el período de septiembre a diciembre de 2017, a través de la aplicación de cuestionario estructurado, que abarcó características sóciodemográficas, estilo de vida, nutrición y factores de riesgo específicos. Para análisis de datos, se utilizó estadística descriptiva, Prueba de Mann Whitney y Anova, considerando nivel de significancia del 5\%, que fue realizado a través del programa Epi Info. Resultados: hubo predominio en el grupo de edad entre 25 a 44 años (34,1\%). La realización de actividad física fue referida por el $36,4 \%$, práctica del tabaquismo por el 10,3\% y la ingestión de bebidas alcohólicas en el $20,3 \%$ de las mujeres. La medida de la circunferencia abdominal se encontraba inadecuada en el 47,9\% de las mujeres. Conclusión: las condiciones de salud de las participantes, relacionadas principalmente con los hábitos de vida, caracterizaron un perfil de riesgo para el desarrollo de enfermedades crónicas no transmisibles. Conocer el perfil de las mujeres atendidas en la ESF contribuye a la planificación de estrategias educativas a esta población.

Descriptores: Perfil de Salud; Salud de la Mujer; Estrategia Salud de la Familia.

\section{INTRODUÇÃO}

Nas últimas décadas, o país passou por grandes transformações socioeconômicas, marcadas

principalmente, pela industrialização, Journal Health NPEPS. 2019 jan-jun; 4(1):242-257. crescimento econômico, urbanização acelerada e pelo estabelecimento de novos padrões de consumo e de modos de se viver, que alteraram diretamente a situação de saúde da população. Os avanços na área médica, na indústria 
farmacêutica, aliados à difusão de métodos contraceptivos e às mudanças do papel feminino na sociedade, resultaram nas reduções das taxas de mortalidade e de natalidade, que se constituíram como indicativos do aumento da expectativa de vida e do envelhecimento populacional, alterando significativamente as necessidades de saúde da população 1 .

Consolidou-se também, mudanças no perfil epidemiológico brasileiro, no passado demarcado pela enorme incidência de doenças infectoparasitárias e, atualmente, este quadro é caracterizado pelo aumento expressivo de Doenças Crônicas Não Transmissíveis (DCNT) e pelo crescimento dos índices de morbimortalidade decorrentes de violência por causas externas ${ }^{2}$. Nesse sentido, as DCNT correspondem a mais de $70 \%$ das causas de morte no Brasil, cujos principais determinantes residem em fatores de risco modificáveis, como inatividade física, dieta não balanceada, obesidade, tabagismo e consumo abusivo de bebidas alcoólicas ${ }^{3}$.

Em relação ao sexo/gênero feminino, que são a maioria da população brasileira, cujas projeções para o ano de 2019 são na ordem de 52.981,934 mulheres, na faixa etária entre 25 a 59 anos $^{4}$ e as principais usuárias do Sistema Único de Saúde $(\mathrm{SUS})^{1}$, observa-se que as principais causas de morte neste grupo são as doenças cardiovasculares, especialmente, o acidente vascular cerebral e infarto agudo do miocárdio; as neoplasias, com destaque para o câncer de mama; doenças do aparelho respiratório e as doenças endócrinas, nutricionais e metabólicas, principalmente, o diabetes mellitus ${ }^{5}$.

Segundo dados da Organização Mundial da Saúde (OMS), as mulheres vivem, em média, de seis a oito anos mais que os homens, porém adoecem com maior frequência, por motivos relacionados, particularmente, à violência doméstica e sexual, e são mais vulneráveis a certas doenças de causas previníveis, como desnutrição e mortalidade materna. Associado a esse quadro, o número de mulheres em situação de pobreza é superior ao de homens, apesar de possuírem uma jornada de trabalho maior, em que metade de seu tempo é destinada as atividades não remuneradas, o que reduz, drasticamente, seu acesso a bens sociais, especialmente os serviços de saúde ${ }^{3}$.

Diante dessa realidade de desigualdades nas relações de poder 
entre 0 sexo/gênero masculino e feminino, que impactam diretamente nas condições de saúde das mulheres ${ }^{1}$, ocorreram mudanças no âmbito das políticas nacionais de saúde das mulheres, antes restritas ao viés materno-infantil e reprodutivo, cujas ações eram baseadas na especificidade biológica e na sua função como cuidadora do lar e da educação dos filhos, deixando-as sem assistência durante a maior parte de suas vidas. Atualmente, essas políticas primam pela busca da integralidade do cuidado ${ }^{6}$.

Nesta perspectiva, tendo em vista que a maior causa de morbimortalidade deste grupo é decorrente de DCNT, as neoplasias representam a segunda causa de óbito em mulheres e dentre estas, o câncer de mama representa o primeiro lugar, cujo diagnóstico tardio ocorre em cerca de $60 \%$ dos $\operatorname{casos}^{7}$. O câncer cervical é o terceiro mais frequente e, praticamente, todos os casos estão associados à infecção genital pelo Papiloma Vírus Humano (HPV) ${ }^{8}$. Observa-se, porém que o maior número de mulheres realizando o exame preventivo para câncer cervical está abaixo dos 35 anos, enquanto o risco para desenvolver da doença aumenta a partir dessa idade ${ }^{3}$.
Além disso, as possibilidades de redução do risco de câncer de mama implicam em mudanças de hábitos, como redução do tabagismo, do uso de álcool, da obesidade e do sedentarismo, fatores que também estão associados ao maior risco de Doença Arterial Coronariana $(\mathrm{DAC})^{5}$. A detecção precoce é fundamental para um melhor prognóstico, tendo-se ampliado, nos últimos anos, a cobertura de exames preventivos para os cânceres de mama e de colo do útero?.

Neste contexto, a atenção integral à saúde da mulher tem como porta de entrada a Estratégia Saúde da Família (ESF), na tentativa de ampliar sua resolutividade na produção do cuidado, que visa à promoção de saúde, autonomia e cidadania da população feminina, admitindo-se o conceito de territorialidade ${ }^{3}$.

Diante do exposto, este estudo objetivou descrever o perfil de saúde de mulheres atendidas em Estratégias Saúde da Família em Mato Grosso.

\section{MÉTODO}

Trata-se de um estudo transversal, descritivo e com abordagem quantitativa, realizado com a população do sexo/gênero feminino adstrita no 
território de abrangência de três Estratégias Saúde da Família, pertencentes ao município de Rondonópolis, estado de Mato Grosso. A cidade localiza-se na região sudeste do estado, distante $200 \mathrm{~km}$ da capital Cuiabá, apresenta aproximadamente, 220 mil habitantes ${ }^{10}$ e possui 35 unidades de ESF cadastradas no Cadastro Nacional de Estabelecimentos de Saúde (CNES) para atender à população. As três ESF foram selecionadas aleatoriamente, por meio de sorteio entre as 35 unidades cadastradas.

A pesquisa foi desenvolvida por acadêmicos dos cursos de Bacharel em Enfermagem e Medicina que fazem parte do Programa de Educação pelo Trabalho(PET- Saúde/GraduaSUS) resultado da parceria entre a Secretaria Municipal de Saúde e a Universidade Federal de Mato Grosso (UFMT), Campus Universitário de Rondonópolis, no período de 2016 a 2018, que visa a qualificação dos processos de integração serviço-ensino-comunidade de forma articulada entre o Sistema Único de Saúde (SUS) e a instituição de ensino.

A coleta de dados ocorreu no período de setembro a dezembro de 2017, durante a realização de visitas domiciliares, por meio de um questionário estruturado, que abrangeu as características sóciodemográficas, estilo de vida, nutrição e fatores de risco específicos; e contendo as seguintes variáveis: faixa etária, realização de atividade física, circunferência abdominal, ingesta de refrigerantes e guloseimas, uso de contraceptivo, tabagismo e etilismo, exames preventivos para câncer de mama (mamografia) e de colo uterino. Tomando-se como variáveis de desfecho, - prognóstico correspondente aos diferentes hábitos de vida das mulheres em questão e a estratégia de rastreamento para diagnóstico precoce dos acometimentos citados.

Adotou-se como critérios de inclusão para participação: a) mulheres com idade igual ou superior a 18 anos; b) moradoras da área de abrangência das ESF participantes; c) e que se encontravam na residência em uma das três visitas, em dias e horários diferentes. Foram excluídos os questionários: a) com informações incompletas; ou b) que cujas mulheres não aceitaram assinar o Termo de Consentimento Livre Esclarecido (TCLE). Cabe ressaltar que a amostra foi do tipo aleatória por conveniência, definida pela facilidade de acesso ao público investigado das três unidades escolhidas para desenvolver o projeto. 
Para elaboração do banco de dados, realizou-se dupla digitação em planilha eletrônica. Na análise de dados, descreveu-se as variáveis por meio de frequência absoluta $e$ frequência relativa. Os valores correspondentes às variáveis de hábitos de vida, obtidas da população feminina, bem como as dos exames preventivos, foram tabulados e submetidos a tratamento estatístico por meio do Teste Mann Whitney e Anova, considerando nível de significância de 5\% $(p<0,05)$, que foram realizados através do programa Epi Info (TM) 3.5.1.

0 estudo atendeu aos aspectos éticos em pesquisa de acordo com a Resolução n. ${ }^{\circ}$ 466/2012. Este foi submetido e aprovado pelo Comitê de Ética em Pesquisa da Universidade Federal de Mato Grosso, Campus Universitário de Rondonópolis, de acordo com o protocolo $n .^{\circ} 2.034 .725$ e CAAE $n^{\circ}$ 62735116.4.0000.8088.

\section{RESULTADOS}

Entre 261 mulheres participantes da pesquisa, 60,5\% ( $n=158)$ pertenciam às faixas etárias de 25 a 64 anos preconizadas pelo Ministério da Saúde para realização do exame de Colpocitopatologia Oncótica (CCO) e $51,3 \%(n=134)$ às faixas etárias de 50 a
59 anos, preconizadas para realização do exame de Mamografia, conforme exposto na Tabela 1.

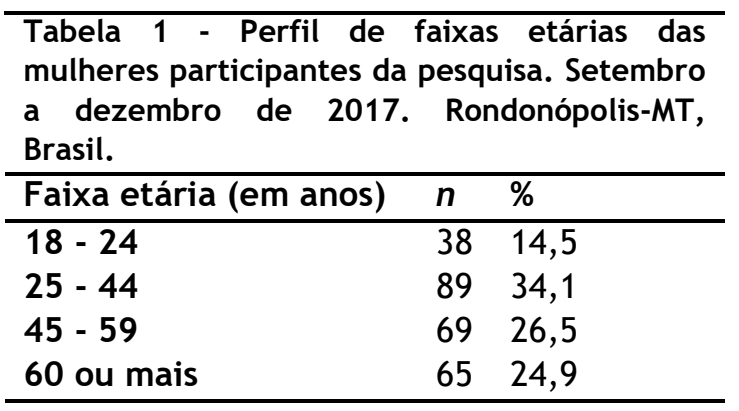

Conforme exposto na Tabela 2, evidencia-se que 48,65\% (n=127) das mulheres nas faixas etárias preconizadas para realização de CCO confirmaram realização do referido exame. Em relação à mamografia, 31,8\% $(n=83)$ das mulheres que se encontravam nas faixas etárias preconizadas responderam afirmativamente quanto à sua realização.

A Tabela 3 expõe os fatores de risco/proteção para câncer de colo do útero elencados durante a entrevista e as frequências de respostas obtidas. Dentre tais fatores, a resposta sim foi predominante somente quanto ao consumo de refrigerantes e guloseimas.

A Tabela 4 denota ausência de associação positiva entre os fatores de risco/proteção para câncer de colo uterino e realização de olpocitopatologio Oncótica, com valores de $\mathrm{p}$ superiores a 0,05 . 
Tabela 2 - Frequência de realização dos exames CCO e mamografia pelas mulheres participantes da pesquisa. Setembro a dezembro de 2017. Rondonópolis - MT, Brasil.

\begin{tabular}{|c|c|c|c|c|c|c|c|c|c|c|c|c|}
\hline \multirow[b]{2}{*}{ Faixa etária } & \multicolumn{6}{|c|}{$\begin{array}{l}\text { Colpocitopatologia } \\
\text { Oncótica (CCO) }\end{array}$} & \multicolumn{6}{|c|}{ Mamografia } \\
\hline & \multicolumn{2}{|l|}{ Sim } & \multicolumn{2}{|c|}{ Não } & \multicolumn{2}{|c|}{$\begin{array}{l}\text { Não } \\
\text { informado }\end{array}$} & \multicolumn{2}{|c|}{ Sim } & \multicolumn{2}{|c|}{ Não } & \multicolumn{2}{|c|}{$\begin{array}{l}\text { Não } \\
\text { Aplicável }\end{array}$} \\
\hline & $\mathrm{n}$ & $\%$ & $\mathrm{n}$ & $\%$ & $\mathrm{n}$ & $\%$ & $\mathrm{n}$ & $\%$ & $\mathrm{n}$ & $\%$ & $\mathrm{n}$ & $\%$ \\
\hline $18-24$ & 26 & 66,7 & 12 & 33,3 & 0 & 0 & 2 & 2,8 & 6 & 16,7 & 30 & 80,6 \\
\hline $25-44$ & 71 & 79,8 & 0 & 0 & 18 & 20,2 & 13 & 14,6 & 18 & 20,2 & 58 & 65,2 \\
\hline $45-59$ & 56 & 81,2 & 0 & 0 & 13 & 18,8 & 45 & 65,2 & 24 & 34,8 & 0 & 0 \\
\hline 60 ou mais & 53 & 81,5 & 12 & 18,5 & 0 & 0 & 38 & 58,5 & 27 & 41,5 & 0 & 0 \\
\hline
\end{tabular}

Tabela 3 - Fatores de risco/proteção relacionados com o câncer de colo do útero das mulheres participantes da pesquisa. Setembro a dezembro de 2017. Rondonópolis - MT, Brasil.

\begin{tabular}{|c|c|c|}
\hline Variável & $\mathbf{n}$ & $\%$ \\
\hline Atividade física & $\mathrm{N}$ & $\%$ \\
\hline $\operatorname{Sim}$ & 95 & 36,4 \\
\hline Não & 159 & 60,9 \\
\hline Não informado & 7 & 2,7 \\
\hline Total & 261 & 100 \\
\hline Circunferência abdominal & $\mathrm{N}$ & $\%$ \\
\hline Adequada & 52 & 19,9 \\
\hline Inadequada & 125 & 47,9 \\
\hline Não informado & 84 & 32,2 \\
\hline Total & 261 & 100 \\
\hline Uso de contraceptivos & $\mathrm{N}$ & $\%$ \\
\hline Sim & 64 & 24,5 \\
\hline Não & 184 & 70,5 \\
\hline Não informado & 13 & 5 \\
\hline Total & 261 & 100 \\
\hline Etilismo & $\mathrm{N}$ & $\%$ \\
\hline Sim & 53 & 20,3 \\
\hline Não & 189 & 72,4 \\
\hline Ex-etilista & 14 & 5,4 \\
\hline Não informado & 5 & 1,9 \\
\hline Total & 261 & 100 \\
\hline Consumo de refrigerantes e guloseimas & $\mathrm{N}$ & $\%$ \\
\hline $\operatorname{Sim}$ & 188 & 72 \\
\hline Não & 71 & 27,2 \\
\hline Não informado & 2 & 0,8 \\
\hline Total & 261 & 100 \\
\hline Tabagismo & $\mathrm{N}$ & $\%$ \\
\hline Sim & 27 & 10,3 \\
\hline Não & 189 & 72,4 \\
\hline Ex-tabagista & 42 & 16,1 \\
\hline Não informado & 3 & 1,1 \\
\hline Total & 261 & 100 \\
\hline
\end{tabular}


Tabela 4 - Associação entre fatores de risco/proteção para câncer de colo uterino e realização de Colpocitopatologia Oncótica. Setembro a dezembro de 2017. Rondonópolis - MT, Brasil.

\begin{tabular}{llll}
\hline Variáveis & Realização do exame & $\begin{array}{l}\text { Médias e } \\
\text { desvios-padrão }\end{array}$ & Valor de p \\
\hline Atividade & Sim & $3,5000 \pm 13,4802$ & 0,2724 * \\
Física & Não & $13,8333 \pm 32,8854$ & \\
Circunferência & Não informado & $1,7097 \pm 0,4614$ & 0,0754 * \\
Abdominal & Adequado & $28,1748 \pm 43,3820$ & \\
& Inadequado & $54,3750 \pm 49,5570$ & 0,4714 * \\
Uso de & Não informado & $48,6774 \pm 49,5314$ & \\
Contraceptivo & Sim & $5,9806 \pm 19,9353$ & 0,1780 * \\
& Não & $9,8333 \pm 27,4664$ & \\
Etilismo & Não informado & $8,0968 \pm 24,2698$ & 0,0875 * \\
& Sim & $2,8252 \pm 9,5585$ & \\
Consumo de & Não & $9,8750 \pm 27,4532$ & \multirow{2}{*}{0,8888 * } \\
refrigerantes e & Não informado & $4,8065 \pm 17,4879$ & \\
guloseimas & Sim & $1,7816 \pm 6,8222$ & \\
& Não & $5,2500 \pm 19,9723$ & $1,1290 \pm 0,3408$ \\
Tabagismo & Não informado & $2,0728 \pm 0,5495$ & \\
& Sim & $10,0833 \pm 27,3892$ & \\
\hline
\end{tabular}

${ }^{*}$ Teste de Mann Whitney.

Tabela 5 indica associação positiva entre os seguintes fatores de risco/proteção para câncer de mama e a realização de mamografia: atividade física, circunferência abdominal, uso de contraceptivos, consumo de refrigerantes e guloseimas e tabagismo, com valores de $p$ inferiores a 0,05.

Tabela 5 - Associação entre fatores de risco/proteção para câncer de mama e realização de mamografia. Setembro a dezembro de 2017. Rondonópolis - MT, Brasil.

\begin{tabular}{llll}
\hline Variáveis & Realização do exame & $\begin{array}{l}\text { Médias e } \\
\text { desvio-padrão }\end{array}$ & Valor de p \\
Atividade Física & Sim & $2,5306 \pm 9,8580$ & \\
& Não & $4,3200 \pm 15,7833$ & 0,0236 * \\
& Não aplicável & $6,0682 \pm 20,4010$ & \\
Circunferência Abdominal & Adequado & $22,6327 \pm 40,0881$ & $0,0155^{* *}$ \\
& Inadequado & $38,0533 \pm 47,3594$ & \\
& Não aplicável & $40,2955 \pm 47,9804$ & $<0,001$ * \\
Uso de contraceptivos & Sim & $8,8673 \pm 25,1280$ & \\
& Não & $9,6000 \pm 26,5427$ & $0,0792 *$ \\
Etilismo & Não aplicável & $1,4773 \pm 0,5023$ & \\
& Sim & $2,9184 \pm 9,8176$ & 0,0138 * \\
Consumo de refrigerantes & Não & $4,4533 \pm 15,7620$ & \\
e guloseimas & Não aplicável & $3,9545 \pm 14,5854$ & $1,3673 \pm 0,4846$ \\
& Sim & $2,5867 \pm 11,2923$ & $0,0598 * *$ \\
Náo & Não aplicável & $2,2727 \pm 10,4362$ & \\
\hline
\end{tabular}

*Teste de Mann Whitney. ** Teste ANOVA

Journal Healtn Nrtrs. LUTy jan-jun; $4(1): \angle 4 \angle-\angle J /$. 


\section{DISCUSSÃO}

O cuidado da mulher no que concerne à saúde reprodutiva tem sido enfatizado pelos sistemas de saúde, com ênfase às seguintes vertentes: pré-natal, parto, puerpério; bem como planejamento no âmbito da reprodução. Outras vertentes priorizadas são: prevenção referente às neoplasias malignas de mama e de colo uterino. Entretanto, a prestação de atendimento às mulheres de forma integral é um processo em fase ainda não concretizada. Este processo engloba acolher requisições e carências do público feminino. Atingir a integralidade no quesito da Atenção Básica envolve o estabelecimento de ações amplas que contemplem a saúde e as necessidades apresentadas pelas usuárias ${ }^{11}$.

A idade é fator de risco para o desenvolvimento de câncer de mama e de colo de útero em mulheres de forma que, com o avanço da idade, as consultas e exames preventivos precisam ser mais frequentes. A realização do exame preventivo de CCO entre as mulheres na faixa 25 e 44 anos, foi de $79,77 \%$ e entre 45 e 59 anos, com $81,15 \%$. A esse respeito, dados se assemelham ao estudo junto a mulheres cadastradas nas Unidades Básicas de
Saúde (UBS) do Município de Porto Velho (RO), em que $82,8 \%(n=227)$ admitiram já ter realizado o exame ${ }^{12}$.

$\mathrm{Na}$ faixa etária de 45 a 59 anos, $65,21 \%$ das pacientes já foram submetidas ao exame de mamografia, enquanto na faixa etária de 60 anos ou mais, $58,46 \%$ já o realizaram. Os dados desta pesquisa diferem de outro estudo ${ }^{13}$ em que se constatou $89,9 \% \quad(n=396)$ das mulheres adultas na faixa etária entre 40 a 59 anos já haviam realizado mamografia em algum momento da vida e em relação às participantes com idade entre 60 a 69 anos, 90,7\% ( $n=439)$ realizaram o mencionado exame. A esse respeito observa-se a diferença no que se refere à maior frequência de realização da mamografia entre as mulheres idosas pelo outro estudo ${ }^{13}$ enquanto no trabalho aqui exposto, tal frequência foi maior nas mulheres com idade entre 45 e 59 anos.

De acordo com as novas diretrizes nacionais, a estratégia de rastreamento recomendado é a realização de mamografia bienal para mulheres entre a faixa etária de 50 a 69 anos, contudo há que se respeitarem os valores e preferências individuais. As estratégias de diagnóstico precoce conduzidas aos casos com sinais e 
sintomas devem complementar o rastreamento ${ }^{14}$.

Quanto à atividade física, 36,4\% das mulheres afirmaram realizá-la, o que difere de outro estudo ${ }^{15}$, cujo objetivo era identificar a prática de atividade física por adultos nas quatro regiões de Brasília - Distrito Federal e concluíram que entre as mulheres, a prática foi correspondente a $79 \%(n=210)$.

Destacam-se os benefícios da atividade física ao grupo feminino, tanto físico como mental, como melhora da autoestima, dos sintomas de ansiedade e depressão, além de contribuir para a melhora da capacidade cognitiva no processo de envelhecimento ${ }^{16}$. A esse respeito, os dados deste estudo são preocupantes, pois menos da metade das mulheres realizam alguma modalidade de atividade física, o que sugere a necessidade de um acompanhamento e estimulação das equipes da ESF para a sua realização.

Em relação à circunferência abdominal 47,9\% apresentavam inadequação. Em pesquisa realizada ${ }^{17}$, ao estimarem a prevalência de adiposidade abdominal em uma população adulta no município de São Francisco do Conde, Bahia, detectaram $76,9 \%(n=302)$ das mulheres com medidas de circunferência da cintura enquadradas nas categorias de risco elevado ou risco muito elevado (parâmetros enfatizados pela Organização Mundial da Saúde), ou seja, predominância da circunferência abdominal inadequada no público feminino, que aumenta o risco para doenças cardiovasculares.

No que se refere ao consumo de refrigerantes e guloseimas entre as participantes, obtendo-se uma frequência relativa de resposta positiva de $72 \%$. Estudos indicam a associação entre a ingesta de refrigerantes ao ganho de peso e desenvolvimento de DCNT $^{18}$. Para tanto, a adoção de estratégias educativas que estimulem a substituição de alimentos com altos teores de açúcares para aqueles saudáveis deve fazer parte das ações de promoção da saúde, dentre as atividades das equipes da ESF.

A prática do tabagismo foi relatada por $10,3 \%$ das mulheres e a ingestão de bebidas alcoólicas em 20,3\%. Em estudo ${ }^{19}$ realizado com o intuito de detectar o nível de desigualdade social presente no contexto de acesso ao exame de citologia oncótica, conforme a posse de plano privado de saúde, bem como outras variáveis de saúde e socioeconômicas no público feminino que residiam na zona urbana do 
município de Campinas, estado de São Paulo, detectaram que em uma amostra de 507 mulheres submetidas ao exame entre os 2005 a 2007, 16,56\% eram fumantes e $30,57 \%$ consumiam bebidas alcoólicas.

Houve associação entre a variável atividade física e a variável realização do exame de mamografia $(p=0,0236)$. Quanto à variável realização do exame de CCO, a associação com a variável atividade física não foi evidenciada $(p=0,2724)$. Embora a herança genética seja um fator importante, um estilo de vida inadequado e o sedentarismo são apontados como os principais fatores de contribuição para o número aumentado de casos referentes a doenças crônicas; abrangendo o diabetes, as doenças cardiovasculares e o câncer. Quanto maior a idade, maior o percentual de influência sobre 0 risco. Aproximadamente entre um quarto e um terço dos casos estão associados a sobrepeso, obesidade e percentuais de gordura centralizada acentuada ${ }^{20}$.

o sedentarismo é um considerável fator de risco para o câncer de mama. Estudos apresentam que mulheres fisicamente ativas possuem risco menor entre $25 \%$ e $30 \%$ na incidência desta doença. Tal redução se refere não somente a mulheres ativas por toda a vida, mas também àquelas que iniciaram a prática de exercícios em períodos mais tardios ${ }^{21}$.

A variável circunferência abdominal apresentou associação com a variável realização de mamografia $(p=0,0155)$ e não apresentou associação com a variável realização do exame de CCO $(p=0,0754)$. Em estudo ${ }^{22}$ houve evidências de que a distribuição da gordura corporal se relaciona positivamente com o risco de câncer de mama, principalmente no período de 15 anos posteriores à menopausa: a cada 10 $\mathrm{cm}$ de aumento na circunferência da cintura, o risco relativo equiparou-se a 1,13 (95\%IC, 1,03-1,24).

0 padrão de obesidade central da gordura está relacionado à maior probabilidade de hipertensão e a níveis sanguíneos tanto de glicose quanto de triglicerídeos aumentados. Pessoas com obesidade central apresentam risco elevado para doenças cardiovasculares e diabetes ${ }^{23}$.

A variável uso de contraceptivos apresentou associação com a variável realização de mamografia $(p=<0,001)$ e não apresentou associação com a variável realização do exame de CCO $(p=0,4714)$. Cabe ressaltar o grupo de maior risco para anticoncepção e câncer 
de mama é aquele que inicia seu uso com baixa idade, por tempo prolongado e antes da primeira gestação ${ }^{24}$, logo, faz-se indispensável que os exames de preventivos e de controle sejam realizados por esta população.

A variável etilismo apresentou associação com a variável realização de mamografia $(p=0,0792)$ e não apresentou associação com a variável realização do exame de CCO $(p=0,1780)$, ocorrendo o mesmo com a variável tabagismo: $p=0,0598$ e $p=0,8888$, respectivamente, assim pode-se dizer que o consumo de álcool é um fator de risco relevante para o câncer de mama ${ }^{25}$. É necessário que estudos epidemiológicos sejam realizados no sentido de clarificar o papel influente do tabagismo em relação ao risco para desenvolvimento de neoplasias. Ainda existem limitações de evidência no que concerne à associação do tabagismo com câncer de mama e leucemia linfocítica aguda ${ }^{26}$.

A variável consumo de refrigerantes e guloseimas apresentou associação com a variável realização de mamografia $(p=0,0138)$ e não apresentou associação com a variável realização do exame de CCO $(p=0,0875)$. Ressalta-se que há estimativas de que 80 a $90 \%$ dos cânceres estão associados a fatores ambientais e, destes, $35 \%$ a dieta ${ }^{27}$.
Neste contexto, o equilíbrio alimentar se apresenta como um fator importante para prevenção do câncer de colo do útero. Contudo, estudos mais abrangentes precisam ser realizados para que recomendações nutricionais específicas e a equipe das ESF proporcionem prevenção frente a estes dados apresentados.

\section{CONCLUSÃO}

Dentre as condições de saúde das mulheres participantes desse estudo, relacionadas principalmente aos hábitos de vida, caracterizaram um perfil de risco para desenvolvimento de DCNT, ao serem encontradas altas taxas de prevalência de sedentarismo, de circunferência abdominal inadequada e de consumo de refrigerantes e guloseimas. Este risco aumenta com o avançar da idade, exigindo que ações de promoção e prevenção à saúde sejam articuladas, como é o caso da mamografia de rastreamento para câncer de mama e do CCO.

A realização da mamografia apresentou correlação positiva com atividade física, circunferência abdominal, consumo de refrigerante e guloseimas, tabagismo e uso de contraceptivos, apontando para a 
importância de uma abordagem multicêntrica para efetividade das ações preventivas. Ressalta-se que, possivelmente, a menor frequência de associações envolvendo a variável realização de exame colpocitopatológico ocorreu por este procedimento ser disponibilizado na Atenção Primária à Saúde, entretanto de forma deficitária.

Diante disso, conhecer o perfil das mulheres atendidas na ESF contribui para o planejamento da gestão do cuidado, englobando estratégias de educação em saúde voltadas à população. Dentre as limitações deste estudo, destaca-se o tamanho da amostra em relação à população do município. Assim, sugere-se novos estudo sobre a temática, a fim de que sejam traçadas ações efetivas no âmbito da atenção primária para este público.

\section{REFERÊNCIAS}

1. Ministério da Saúde (BR). Secretaria de Políticas para as Mulheres. Plano Nacional de Políticas para as Mulheres 2013 - 2015. Brasília: Ministério da Saúde; 2013.

2. Silva SF. Organização de redes regionalizadas e integradas de atenção à saúde: desafios do Sistema
Único de Saúde (Brasil). Ciênc saúde colet. $2011 ; 16(6): 2753-62$.

3. Ministério da Saúde (BR). Secretaria de Atenção à Saúde, Departamento de Ações Programáticas Estratégicas. Política Nacional de Atenção Integral à Saúde da Mulher. Brasília: Ministério da Saúde; 2011.

4. IBGE. Instituto Brasileiro de Geografia e Estatística. Projeções da população por sexo e idade 20102060. 2018. Disponível em: https://www.ibge.gov.br/estatistica S-

novoportal/sociais/populacao/9109projecao-dapopulacao.html?=\&t=resultados.

5. Sales MC, Rocha ACD, Pedraza DF. Características sóciodemográficas e fatores importantes para a manutenção da saúde em mulheres. Rev ras promoç sabúde. 2014; 27(40):503-12.

6. Freitas GL, Vasconcelos, CTM, Moura ERF, Pinheiro, AKB. Discutindo a política de atenção à saúde da mulher no contexto da promoção da saúde. Rev eletrônica enferm. 2009; 11(2):424-28.

7. Instituto Nacional de Câncer José Alencar Gomes da Silva - INCA. Tipos de câncer: colo de útero. 2018. Disponível em: 
<http://www2.inca.gov.br/wps/wc $\mathrm{m} /$ connect/tiposdecancer/site/hom e/colo_utero/definicao>.

8. Ministério da Saúde (BR). Secretaria de Vigilância em Saúde. Departamento de Análise de Situação de Saúde. Plano de ações estratégicas para o enfrentamento das doenças crônicas não transmissíveis (DCNT) no Brasil 20112022. Brasília: Ministério da Saúde; 2011.

9. Organização Mundial a Saúde - OMS. Mulheres e saúde: evidências de hoje, agenda de amanhã. 2011. 112p. Disponível em: https://www.who.int/ageing/mulhe res_saude.pdf

10. IBGE. Instituto Brasileiro de Geografia e Estatística. Diretoria de Pesquisas, Coordenação de População e Indicadores Sociais, Estimativas da população residente com data de referência 10 de julho de 2018. Disponível em: https: //cidades.ibge.gov.br/brasil/ $\mathrm{mt} /$ rondonopolis/panorama

11. Ministério da Saúde (BR). Protocolos da Atenção Básica: Saúde das Mulheres. Brasília: Ministério da Saúde; 2016.

12. Lucena LT, Zãn DG, Crispim PTB, Ferrari JO. Fatores que influenciam a realização do exame preventivo do câncer cérvico-uterino em Porto Velho, Estado de Rondônia, Brasil. Rev Pan-Amaz Saúde. 2011; $2(2): 45-50$.

13. Schneider IJC, Giehl MWC, Boing AF, D’Orsi E. Rastreamento mamográfico do câncer de mama no Sul do Brasil e fatores associados: estudo de base populacional. Cad Saúde Pública. 2014 set; 30(9):1987-97.

14. Migowski A, Silva GA, Dias MBK, Diz MDPE, Sant'Ana DR, Nadanovsky P. Diretrizes para detecção precoce do câncer de mama no Brasil. II Novas recomendações nacionais, principais evidências e controvérsias Cad Saúde Pública. 2018; 34(6):e00074817.

15. Thomaz PMD, Costa THM, Silva EF, Hallal PC. Factors associated with physical activity in adults in Brasília, Central-West Brazil. Rev Saúde Pública. 2010; 44(5):894-900.

16. Gonçalves AKS, Canário ACG, Cabral PUL, Silva RAH, Spyrides MHC, Giraldo PC, Eleutério Jr J. Impact of physical activity on quality of life in middle-aged women: a population based study. Rev Bras Ginecol Obstet. 2011; 33(12):408-13. 
17. Oliveira LC, West LEM, Araújo EA, Sobrinho CLN. Prevalência de adiposidade abdominal em adultos de São Francisco do Conde, Bahia, Brasil, 2010. Epidemiol Serv Saúde. 2015; 24(1):135-144.

18. Ribeiro THT, Albuquerque TG, Silva DL, Oliveira KCS, Filgueiras $\mathrm{N}$, Mendes VS, et al. Revisão bibliográfica: consumo de refrigerantes associado à obesidade. Adolesc Saúde. 2012; 9(4):44-48.

19. Amorim VMSL, Barros MBA. Equidade no acesso ao exame de Papanicolaou: estudo de base populacional no município de Campinas, São Paulo, Brasil. Rev Bras Epidemiol. 2014; 17 suppl 2:136-149.

20. Friedenreich $C M$, Orenstein MR. Physical activity and cancer prevention: etiologic evidence and biological mechanisms. Am soc nutr sci. 2002; 132(11 suppl):3456S$3456 \mathrm{~S}$.

21. Friedenreich CM, Cust AE. Physical activity and breast cancer risk: impact of timing, type and dose of activity and population subgroup effects. $\mathrm{Br} \mathrm{j}$ sports med. 2008; 42:636-647.
22. Macinnis RJ, English DR, Gertig DM, Hopper JL, Giles GG. Body size and composition and risk of postmenopausal breast cancer. Cancer Epidemiol Biomarkers Prev. 2004; 13(12):2117-25.

23. Navarro AM, Stedille MS, Unamuno MRDL, Marchini JS. Distribuiçãa da gordura corporal em pacientes com e sem doenças crônicas: uso da relação cintura-quadril e do índice de gordura do braço. Rev Nutr. 2001; 14(1):37-41.

24. Schunemann Júnior E, Souza RT, Dória MT. Anticoncepção hormonal e câncer de mama. FEMINA. 2011; 39(4):231-35.

25. Wünsch Filho V. Consumo de bebidas alcoólicas e risco de câncer. Rev USP. 2012-2013; 96:3746.

26. Wünsch Filho $V$, Mirra AP, López RVM, Antunes LF. Tabagismo e câncer no Brasil: evidências e perspectivas. Rev Bras Epidemiol. 2010; 13(2):175-87.

27. Hyppolito KPP, Ribeiro KAR. Importância da nutrição na prevenção e no tratamento de neoplasias. Interciência soc. 2014; $3(2): 51-59$. 
Conflito de interesses: Os autores declaram não haver conflito de interesses.

\section{Participação dos autores:}

- Concepção: Silva NR, Xavier HR, Rocha TL, Santos VLT, Mattos M, Santos DAS, Silva Júnior AJ.

- Desenvolvimento: Silva NR, Xavier HR, Rocha TL, Santos VLT, Mattos M, Santos DAS, Silva Júnior AJ.

- Redação e revisão: Silva NR, Xavier HR, Rocha TL, Santos VLT, Mattos M, Santos DAS, Silva Júnior AJ.

Como citar este artigo: Silva NR, Xavier HR, Rocha TL, Santos VLT, Mattos M, Santos DAS, et al. Perfil de saúde de mulheres atendidas em estratégias saúde da família em Mato Grosso. J Health NPEPS. 2019; $4(1): 242-257$.

Submissão: $10 / 02 / 2019$

Aceito: $26 / 05 / 2019$

Publicado: 01/06/2019 